\title{
Automatic strategy to identify the risk of malnutrition in primary care by means of Controlling Nutritional (CONUT) Score: a large population study.
}

\author{
María Salinas ( $\sim$ salinas_mar@gva.es ) \\ Hospital Universitari Sant Joan d'Alacant https://orcid.org/0000-0003-3420-5509 \\ Maite López-Garrigós \\ Hospital Universitari Sant Joan d'Alacant
}

Emilio Flores

Hospital Universitari Sant Joan d'Alacant

Carmen Puche

Hospital Universitari Sant Joan d'Alacant

\section{Pablo Leiva-Salinas}

Universidad Miguel Hernandez de Elche Facultad de Medicina

\section{Ana Santo-Quiles}

Hospital Universitari Sant Joan d'Alacant

Carlos Leiva-Salinas

Department Radiology, University of Missouri

\section{Research article}

Keywords: Risk of Malnutrition, Laboratory Organization and Management, CONUT, Albumin, Strategy

Posted Date: May 27th, 2020

DOI: https://doi.org/10.21203/rs.3.rs-28355/v1

License: (9) This work is licensed under a Creative Commons Attribution 4.0 International License. Read Full License 


\section{Abstract \\ Background}

Risk of malnutrition can be evaluated through Controlling Nutritional (CONUT) Score based on total peripheral lymphocytes, serum albumin, and total cholesterol. Our aim was to automatically calculate CONUT in primary care when involved markers were requested and compare its performance with albumin to identify patients at risk of malnutrition. CONUT was evaluated according to patient age, and calculated the potential expenses to calculate CONUT, when serum markers were no requested, and measured.

\section{Methods}

We calculated CONUT when the three laboratory markers were requested, compared CONUT and albumin, considering risk of malnutrition CONUT $\geq 2$, and albumin $<35 \mathrm{~g} / \mathrm{L}$, and counted the number of albumin and/or cholesterol tests that would have been measured to calculate CONUT in patients with total lymphocytes and serum availability, and calculated hypothetical cost.

\section{Results}

of the 74743 requests from primary care, CONUT was calculated in 9353 patients. $2256(24.1 \%)$ showed risk of malnutrition according CONUT; $630(6.8 \%)$ through albumin. Albumin alone identified $17.5 \%$ of mild cases based on CONUT, but every severe case. The risk of malnutrition increased with patient age. In one year, through an expense of $5430 €$ in measuring the serum non requested test, we could have screened for risk of malnutrition the entire primary care population that underwent laboratory testing.

\section{Conclusion}

CONUT score calculated at no cost when requested involved laboratory markers, detected more patients at risk of malnutrition than albumin, increased with patient age, and could be calculated in all primary care patients by measuring unrequested tests, at a very affordable cost.

\section{Background}

Malnutrition is an unfavorable prognostic factor associated with increased morbidity and mortality, known to increase hospital stays, readmissions, and resources consumption (1).

Albumin, the most extensively studied protein to identify patients at risk of malnutrition, is a useful indicator of general nutrition status for older adults in non-acutely ill states (2). Nutritional status evaluated through NUTritional COntrol (CONUT) based on total peripheral lymphocytes, serum albumin 
and total cholesterol has been shown as a valuable tool for the early detection and monitoring of clinical under nutrition. Since its introduction in 2002 (3), its main application has been to evaluate and quantify the prevalence of malnutrition at hospital admission (4). Since then, the CONUT score has been extensively studied as an efficient tool in different types of patients and contexts and the results in different clinical scenarios have been of most relevance (5-18).

All the above research was conducted in hospitalized patients, and no meaningful information regarding the use of CONUT in primary care is available.

However, it is crucial to look for the feasibility of implementing routine nutritional screening in general practices (19).

Our aim was to automatically calculate the CONUT score in a large number of primary care patients when all three involved laboratory markers were requested, and compare its performance with albumin to identify risk of malnutrition. We planned to evaluate CONUT according to patient age, and evaluate the costs of a potential strategy involving CONUT as a screening tool in all primary care patients that had a laboratory request.

We hypothesized that CONUT score could be a valid first line tool to screen for patients at risk of malnutrition in primary care to later be confirmed / discarded with proven nutrition evaluation tools.

\section{Methods}

\section{Setting and subjects}

The clinical laboratory is located in a 370-bed suburban University Community Hospital that serves the population of the Health Department (HD) (234551 inhabitants). It receives samples from inpatients and outpatients and primary care patients that are phlebotomized in nine different primary care centres. Samples are transported by couriers to the laboratory reception desk. Requests are made by general practitioners (GPs) via computerized order entry, and results are also automatically sent to the electronic medical record. In this study, we included all community inhabitants over 18 years old within the HD covered by the clinical laboratory. The study was approved by the Hospital Research Committee.

\section{Study Design}

A cross sectional study was designed from January 1st 2018 to December 31st 2018. In consensus with GPs, the CONUT score was automatically calculated by our Laboratory Information System (LIS) for every primary care request when the three involved laboratory markers were requested, in accordance with the tool described in Table 1.

\section{Laboratory methods}

The blood samples were collected using BD vacutainer tubes (Beckton Dickinson, NJ, USA). 
Albumin was measured through an immunoturbidimetric assay and total cholesterol through an enzymatic colorimetric method (Cobas 8000 , Roche, Mannheim, Germany).

Total lymphocyte were counted with clinical hematology analyzers (Sysmex, Kobe, Japan).

\section{Data collection}

For all primary care requests, we collected patient's demographic data, CONUT and albumin value from the LIS (iGestlab ${ }^{\circledR}$ ), and compared CONUT and albumin values to evaluate for concordance, considering risk of malnutrition CONUT $\geq 2$, and albumin $<35 \mathrm{~g} / \mathrm{L}$.

We classified results by patient's age, and compared them for the different age brackets.

For the potential strategy involving CONUT as a screening tool in all laboratory requests from primary care, we counted the number of non-demanded albumin and/or total cholesterol, when measured total number of lymphocytes and serum availability. We calculated the hypothetical economic cost based on both tests prices ( $0.09 €$ for total cholesterol, and $0.08 €$ for albumin).

\section{Statistical analysis}

Demographic summary statistics are reported as median (IQR) for continuous and frequency (\%) for categorical variables. Together with the requests' basic information, differences were assessed using Chisquare, U-Mann Whitney test and Kruskal-Wallis test where applicable. A two-sided $p \leq 0.05$ rule was utilized as the criterion for rejecting the null hypothesis of no difference. Statistical anlyses were done using Statistical Package for the Social Sciences (SPSS), Version 22 (IBM Corp., Chicago, Illinois, USA).

\section{Results}

During the study period, the clinical laboratory received 74743 requests from primary care. CONUT score was calculated in 9353 patients, as the 3 involved markers were requested, with a median (IQR) age of 68 (54-82) and $35.6 \%$ of whom were men. Of those, 2256 (24.1\%) showed risk of malnutrition according to the CONUT score (CONUT score $\geq 2$ ), and $630(6.7 \%)$ based on albumin (albumin $<35 \mathrm{~g} / \mathrm{L}$ ). Table 2 shows their demographic information and also albumin values according to CONUT score. Higher CONUT scores were significantly associated with male sex $(P<.05)$, older age $(P<.05)$ and lower albumin value $(\mathrm{P}<.05)$.

As shown in Fig. 1, through both markers, the risk of malnutrition, increased as did patient age.

$100 \%$ was the ability of albumin alone to identify cases of severe malnutrition, but only $17.5 \%$ of mild based on CONUT score (Fig. 2).

From the 74743 total patients, in 65390 the score could have been calculated, as they had total peripheral lymphocytes, and also serum availability to measure the missing non-requested test, either cholesterol or albumin. The missing test was total cholesterol in 3351 and albumin in 64107. Through an expense of 
$5430 €$, the laboratory could have screened the entire HD primary care population that underwent a laboratory test.

\section{Discussion}

This is the first study that investigates the relationship between CONUT, albumin and the risk of malnutrition in primary care. The CONUT score, based on three widely used laboratory markers, detected more patients at risk of malnutrition, than albumin alone. The intervention to automatically report the CONUT score when all three necessary laboratory tests were requested was successful, and at a zero cost. The number of patients at risk of malnutrition through the CONUT score increased with patient age. By measuring non-requested albumin and/or total cholesterol tests needed for CONUT calculation when serum was available and lymphocytes counted, it would be possible to assess the risk of malnutrition of all primary care patients undergoing laboratory testing, at a very affordable and reasonable cost, for later to be confirmed/discarded with proven nutritional tools.

When comparing serum albumin levels $<35 \mathrm{~g} / \mathrm{L}$ as the unique marker for risk malnutrition with CONUT score, it would result in up to $80 \%$ of the subjects being misdiagnosed (20), as albumin alone identified every severe case, but missed a significant number of patients at risk of malnutrition (mild and moderate cases) through CONUT score. However, CONUT score identified the risk of malnutrition in $24.1 \%$ of the studied patients, figures that may seem too high for a primary care population. It might be explained for two facts. First, the patients with the three involved markers, and consequently evaluated for risk of malnutrition through both methods, had a median (IQR) age of 68 . These numbers are not representative of a primary care population but of a primary care elderly population. Second, there could be patients with clinical suspicion of risk of malnutrition, since the GP ordered serum albumin. Consequently, in our study, CONUT score could have been calculated in a primary care elderly population at risk of malnutrition.

Moreover, and to try to explain the differences in the number of patients identified at risk of malnutrition through CONUT and serum albumin, it has been reported that serum albumin cutoff of $35 \mathrm{~g} / \mathrm{L}$ underdiagnoses malnutrition as defined by validated nutrition screening tools such as Mini Nutritional Assessment (MNA), Nutritional Risk Screening 2002 (NRS-2002), Mini Nutritional Assessment-Short Form (MNA-SF), and Geriatric Nutritional Risk Index (GNRI)) (21). Moreover, the utility of serum albumin and the traditional cutoff $(35 \mathrm{~g} / \mathrm{l})$ in physically impaired elderly older people has also been reported that is questionable (22). Based on the above, it might be reasonable to replace albumin for CONUT score. It could be a better laboratory indicator to identify patients at risk of malnutrition, especially key in overcrowded primary care. Malnutrition will be confirmed/discarded as soon as possible with proven nutritional assessment tools, to establish the appropriate measures for its correction and subsequent monitoring.

Our study shows that the CONUT score increased with aging, as we detected more subjects at risk of malnutrition in the elderly population. Malnutrition remains a significant and highly prevalent public 
health problem of developed countries, especially in old population. Earlier identification and appropriate nutrition support may help to reverse or halt the malnutrition trajectory and the negative outcomes associated with poor nutritional status (23). Healthcare professionals should identify the malnutrition risk, and take action, as early as possible (24).

Detecting malnutrition in the general population through traditional tools could be too expensive. Our study results of the potential intervention involving CONUT as a screening tool in all primary care patients who had a laboratory request, by measuring serum albumin and/or total cholesterol when not requested, taking advantage of the serum sample availability and the affordable reagent cost, seems cost-effective. Especially if considering the examined population of more than 70000 primary care patients. The results of our study provide us the opportunity to develop a large-scale opportunistic detection of the risk of malnutrition in the general population, to be subsequently confirmed/discarded through traditional malnutrition evaluation tools.

The study had some limitations. First, patients with CONUT score showing risk of malnutrition were not studied with the nutrition traditional tools, however it has been confirmed in different settings the validity of the CONUT system for early detection and monitoring of clinical undernutrition (25). Second, the calculated economic investments may not apply to other countries or settings, since our laboratory belongs to the Public Health Network, where reagent prices tend to be low.

CONUT score, calculated at no cost when requested involved laboratory markers, detected more patients at risk of malnutrition than albumin, increased with patient age, and could be calculated in all primary care patients by measuring unrequested tests, at a very affordable cost. The early detection of risk of malnutrition at initial stages through the CONUT score, that could be confirmed/discarded through traditional nutritional tools, could greatly improve primary care patient's outcome.

\section{Conclusions}

CONUT score calculated at no cost when requested involved laboratory markers, detected more patients at risk of malnutrition than albumin, increased with patient age, and could be calculated in all primary care patients by measuring unrequested tests, at a very affordable cost.

\section{Abbreviations}

CONUT

Nutritional Control

GNRI

Geriatric Nutritional Risk

GPs

General practitioners

HD 
Health Department

LIS

Laboratory Information System

MNA

Mini Nutritional Assessment

MNA-SF

Mini Nutritional Assessment-Short Form

SPSS

Statistical Package for the Social Sciences

\section{Declarations}

- Ethics approval and consent to participate: Written consent was obtained, the study was approved by the Research Committee of the Hospital Universitario San Juan de Alicante.

- Consent for publication: Not applicable

- Availability of data and materials: The datasets used and/or analysed during the current study are available from the corresponding author on request.

- Competing interests: The authors declare that they have no competing interests

- Funding: This work did not receive any funding.

- Authors' contributions: All authors researched literature and conceived the study. MLG, EF, CP, ASQ and PLS were involved in protocol development and data analysis. MS wrote the first draft of the manuscript. CLS reviewed and edited the manuscript. All authors read and approved the final manuscript.

- Acknowledgements: The authors would like to express their deep gratitude to all the clinical laboratory staff.

\section{References}

1. Lobo Támer G, Ruiz López MD, Pérez de la Cruz AJ. [Hospital malnutrition: relation between the hospital length of stay and the rate of early readmissions]. Med Clin (Barc) [Internet]. 2009 Mar 21 [cited 2019 Feb 8];132(10):377-84. Available from: https://linkinghub.elsevier.com/retrieve/pii/S0025775308000547.

2. Cabrerizo S, Cuadras D, Gomez-Busto F, Artaza-Artabe I, Marín-Ciancas F, Malafarina V. Serum albumin and health in older people: Review and meta analysis. Maturitas [Internet]. 2015 May [cited 2019 Jan 24];81(1):17-27. Available from:

https://linkinghub.elsevier.com/retrieve/pii/S0378512215000572. 
3. de Ulíbarri Pérez JI, González-Madroño Giménez A, González Pérez P, Fernández G, Rodríguez Salvanés F, Mancha Alvarez-Estrada A, et al. [New procedure for the early detection and control of hospital malnutrition]. Nutr Hosp [Internet]. [cited 2019 Jan 24];17(4):179-88. Available from: http://www.nutricionhospitalaria.com/pdf/3323.pdf.

4. Ignacio de Ulíbarri J, González-Madroño A, de Villar NGP, González P, González B, Mancha A, et al. CONUT: a tool for controlling nutritional status. First validation in a hospital population. Nutr Hosp [Internet]. [cited 2019 Jan 28];20(1):38-45. Available from: http://scielo.isciii.es/pdf/nh/v20n1/original1.pdf.

5. Nishikawa H, Yoh K, Enomoto H, Ishii N, Iwata Y, Takata R, et al. The Relationship between Controlling Nutritional (CONUT) Score and Clinical Markers among Adults with Hepatitis C Virus Related Liver Cirrhosis. Nutrients [Internet]. 2018 Aug 29 [cited 2019 Jan 28];10(9):1185. Available from: http://www.ncbi.nIm.nih.gov/pubmed/30158477.

6. Kang HW, Seo SP, Kim WT, Yun SJ, Lee S-C, Kim W-J, et al. Prognostic Impact of Nutritional Status Assessed by the Controlling Nutritional Status (CONUT) Score in Patients with Surgically Treated Renal Cell Carcinoma. Nutr Cancer [Internet]. 2018 Aug 18 [cited 2019 Jan 28];70(6):886-94. Available from: http://www.ncbi.nlm.nih.gov/pubmed/30235010.

7. Iseki Y, Shibutani M, Maeda K, Nagahara H, Ohtani H, Sugano K, et al. Impact of the Preoperative Controlling Nutritional Status (CONUT) Score on the Survival after Curative Surgery for Colorectal Cancer. Chu P-Y, editor. PLoS One [Internet]. 2015 Jul 6 [cited 2019 Jan 28];10(7):e0132488. Available from: https://www.ncbi.nlm.nih.gov/pmc/articles/PMC4492767/.

8. http://doi.wiley.com/10.1111/j.1872-034X.2012.01055.x

Taniguchi E, Kawaguchi T, Otsuka M, Uchida Y, Nagamatsu A, Itou M, et al. Nutritional assessments for ordinary medical care in patients with chronic liver disease. Hepatol Res [Internet]. 2013 Feb [cited 2019 Jan 28];43(2):192-9. Available from: http://doi.wiley.com/10.1111/j.1872-034X.2012.01055.x.

9. Narumi T, Arimoto T, Funayama A, Kadowaki S, Otaki Y, Nishiyama S, et al. Prognostic importance of objective nutritional indexes in patients with chronic heart failure. J Cardiol [Internet]. 2013 Nov [cited 2019 Jan 28];62(5):307-13. Available from: https://linkinghub.elsevier.com/retrieve/pii/S0914508713001688.

10. Solórzano-Pineda OM, Rivera-López FA, Rubio-Martínez B. [Malnutrition incidence in surgical diabetic and non diabetic patients in general surgery department]. Nutr Hosp [Internet]. [cited 2019 Jan 28];27(5):1469-71. Available from: http://scielo.isciii.es/scielo.php?script=sci_arttext\&pid=S021216112012000500015.

11. Wang X-B, Chen J, Xiang B-D, Wu F-X, Li L-Q. High CONUT score predicts poor survival and postoperative HBV reactivation in HBV-related hepatocellular carcinoma patients with low HBV-DNA levels. Eur J Surg Oncol [Internet]. 2018 Nov 10 [cited 2019 Jan 28]; Available from: https://www.ejso.com/article/S0748-7983(18)31998-X/fulltext.

12. Kato $Y$, Yamada S, Suenaga $M$, Takami H, Niwa $Y$, Hayashi $M$, et al. Impact of the Controlling Nutritional Status Score on the Prognosis After Curative Resection of Pancreatic Ductal 
Adenocarcinoma. Pancreas [Internet]. 2018 Aug [cited 2019 Jan 28];47(7):823-9. Available from: http://insights.ovid.com/crossref?an=00006676-201808000-00005.

13. Kono T, Sakamoto K, Shinden S, Ogawa K. Pre-therapeutic nutritional assessment for predicting severe adverse events in patients with head and neck cancer treated by radiotherapy. Clin Nutr [Internet]. 2017 Dec [cited 2019 Jan 28];36(6):1681-5. Available from: https://linkinghub.elsevier.com/retrieve/pii/S0261561416313139.

14. Liang R-F, Li J-H, Li M, Yang Y, Liu Y-H. The prognostic role of controlling nutritional status scores in patients with solid tumors. Clin Chim Acta [Internet]. 2017 Nov [cited 2019 Jan 28];474:155-8. Available from: https://linkinghub.elsevier.com/retrieve/pii/S0009898117303777.

15. La Rovere MT, Maestri R, Olmetti F, Paganini V, Riccardi G, Riccardi R, et al. Additional predictive value of nutritional status in the prognostic assessment of heart failure patients. Nutr Metab Cardiovasc Dis [Internet]. 2017 Mar [cited 2019 Jan 28];27(3):274-80. Available from: https://linkinghub.elsevier.com/retrieve/pii/S0939475316301648.

16. Iwakami N, Nagai T, Furukawa TA, Sugano Y, Honda S, Okada A, et al. Prognostic value of malnutrition assessed by Controlling Nutritional Status score for long-term mortality in patients with acute heart failure. Int J Cardiol [Internet]. 2017 Mar 1 [cited 2019 Jan 28];230:529-36. Available from: https://linkinghub.elsevier.com/retrieve/pii/S0167527316345521.

17. $10.1007 / \mathrm{s} 00384-016-2668-5$

Tokunaga R, Sakamoto Y, Nakagawa S, Ohuchi M, Izumi D, Kosumi K, et al. CONUT: a novel independent predictive score for colorectal cancer patients undergoing potentially curative resection. Int J Colorectal Dis [Internet]. 2017 Jan 10 [cited 2019 Jan 28];32(1):99-106. Available from: http://link.springer.com/10.1007/s00384-016-2668-5.

18. 10.1007/s00268-016-3549-3

Yoshida N, Baba Y, Shigaki H, Harada K, Iwatsuki M, Kurashige J, et al. Preoperative Nutritional Assessment by Controlling Nutritional Status (CONUT) is Useful to estimate Postoperative Morbidity After Esophagectomy for Esophageal Cancer. World J Surg [Internet]. 2016 Aug 24 [cited 2019 Jan 28];40(8):1910-7. Available from: http://link.springer.com/10.1007/s00268-016-3549-3.

19. Hamirudin AH, Charlton K, Walton K, Bonney A, Potter J, Milosavljevic M, Hodgkins A, Albert G, Ghosh A, Dalley A. Feasibility of implementing routinenutritional screening for older adults in Australian general practices: amixed-methods study. BMC Fam Pract. 2014 Nov 25;15:186. doi: 10.1186/s12875-014-0186-5. PubMed PMID: 25421546; PubMed Central PMCID:PMC4247715.

20. Bharadwaj S, Ginoya S, Tandon P, Gohel TD, Guirguis J, Vallabh H, et al. Malnutrition: Laboratory markers vs nutritional assessment. Gastroenterology Report. 2016.

21. Zhang Z, Pereira SL, Luo M, Matheson EM. Evaluation of Blood Biomarkers Associated with Risk of Malnutrition in Older Adults: A Systematic Review and Meta-Analysis. Nutrients [Internet]. 2017 Aug 3 [cited 2019 Jan 28];9(8):829. Available from: http://www.mdpi.com/2072-6643/9/8/829.

22. Kuzuya M, Izawa S, Enoki H, Okada K, Iguchi A. Is serum albumin a good marker for malnutrition in the physically impaired elderly? Clin Nutr. 2007. 
23. Guyonnet S, Rolland Y. Screening for Malnutrition in Older People. Clin Geriatr Med. 2015 Aug;31(3):429-37.

24. http://doi.wiley.com/10.1111/jan.13297

Bauer S, Halfens RJG, Lohrmann C. Changes in nutritional status in nursing home residents and associated factors in nutritional status decline: a secondary data analysis. J Adv Nurs [Internet]. 2017 Oct [cited 2019 Jun 21];73(10):2420-9. Available from:

http://doi.wiley.com/10.1111/jan.13297.

25. González-Madroño A, Mancha A, Rodríguez FJ, Culebras J, de Ulibarri Jl. Confirming the validity of the CONUT system for early detection and monitoring of clinical undernutrition: comparison with two logistic regression models developed using SGA as the gold standard. Nutr Hosp. 2012 MarApr;27(2):564 - 71 Available from: http://scielo.isciii.es/pdf/nh/v27n2/33_original_23.pdf.

\section{Tables}

Due to technical limitiations, Tables 1-2 are provided in the Supplementary Files section.

\section{Figures}

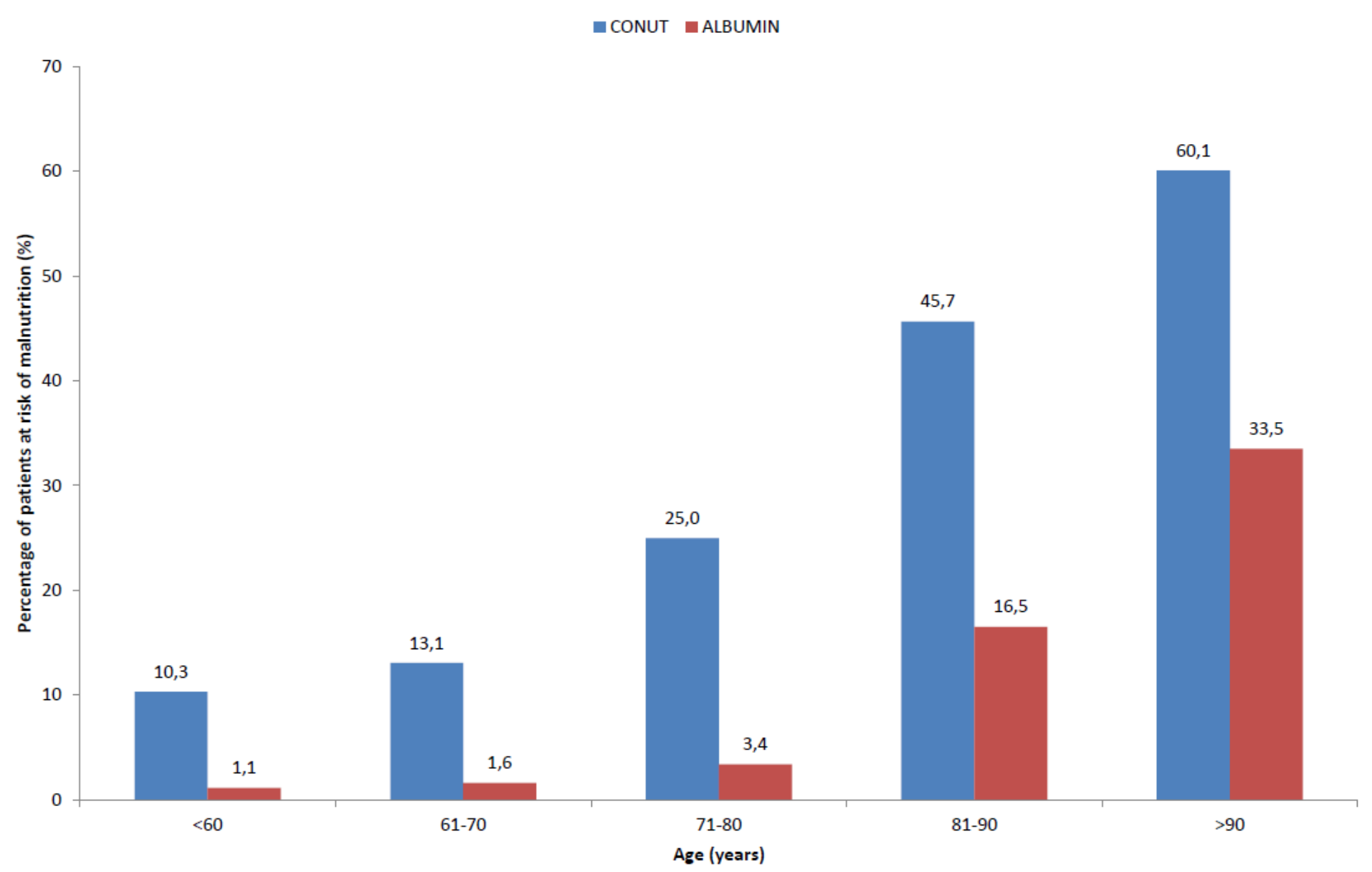


Figure 1

Requests found to have CONUT and albumin values showing risk of malnutrition

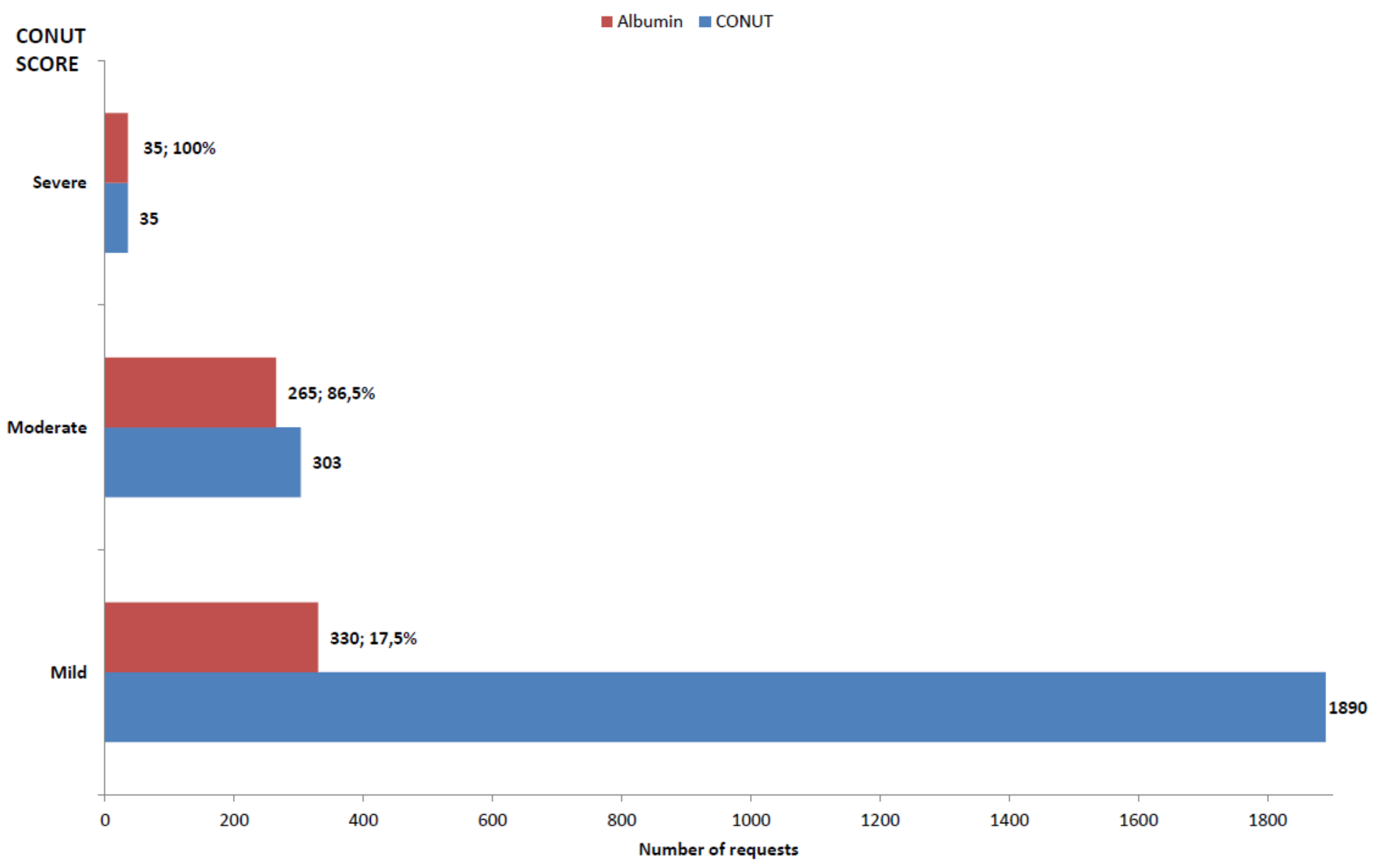

Figure 2

Concordance in the detection of risk of malnutrition through CONUT and albumin

\section{Supplementary Files}

This is a list of supplementary files associated with this preprint. Click to download.

- ETHICSAPPROVAL.pdf

- Table2CONUT.pdf

- Table1CONUT.pdf 